Ann. Sci. forest., 1978, 35 (4), 299-320.

\title{
Aspects de la notion de densité et croissance des arbres en peuplement
}

\author{
J. M. OTTORINI \\ Station de Sylviculture ef de Production, \\ Centre national de Recherches forestières, I.N.R.A., \\ Champenoux, 54280 Seichamps
}

\section{Résumé}

De nombreux travaux de recherche publiés depuis le début des années soixante par des forestiers américains aux U.S.A. et au Canada ont été motivés par l'idée qu'une expression convenable de la densité d'un peuplement pourrait servir de façon non négligeable les études de production et de croissance.

La mesure la plus souvent utilisée, le CCF, a pu avoir plusieurs applications dans divers domaines tels que les normes de peuplements, la détermination de la productivité réelle d'une station pour une essence, ou la protection d'une espèce de sapin attaquée par un scolyte en peuplement mélangé.

En relation étroite avec les mesures de densité de peuplement, les indices de concurrence doivent être considérés comme des mesures locales de densité ; ils ont depuis longtemps été étudiés aux U.S.A., mais ont trouvé récemment leur meilleure expression, et démontré leur efficacité dans l'élaboration de modèles de croissance où l'arbre est l'unité de croissance.

Chaque fois, pour les mesures de densité de peuplement ou les indices de concurrence, il s'est avéré que des informations circonstanciées sur la croissance et le développement des arbres apportent beaucoup aux études de croissance et de production des peuplements.

Les travaux présentés dans cet article ne sont jamais purement théoriques, et sont tous basés sur des données expérimentales. Les raisonnements sont généralement plutôt déductifs qu'inductifs.

\section{1. - Introduction}

Dans l'étude des peuplements forestiers la notion de «densité » constitue toujours une source de difficultés lorsqu'il faut en donner une définition formelle en des termes se rapportant correctement aux phénomènes de la croissance.

On peut penser que résoudre convenablement les problèmes qui se posent alors pourrait conduire en retour à une meilleure compréhension de ces phénomènes et permettre grâce à cela d'en avoir un contrôle plus précis. Ces problèmes qui seront détaillés constituent le vrai sujet de cet article, et ils seront discutés à travers une revue bibliographique.

Les forestiers Européens ont abordé cette question en cherchant à caractériser l'intensité d'une éclaircie. L'article de Hart (1928) est la référence généralement citée, et d'autres définitions ont été proposées après la sienne $\left.{ }^{1}\right)$. efforts.

(1) Voir par exemple : Hummel (1954), Pardé (1961), Vezina (1963), où se trouvent d'excellents aperçus de ces 
Ces recherches en Europe paraissent avoir été délaissées au début des années soixante, sans toutefois être parvenues à une conclusion. Elles se sont cependant poursuivies aux U.S.A. et au Canada où de nombreux chercheurs ont continué à s'y intéresser en leur donnant beaucoup d'importance.

Pour ceux-ci, de plus, la notion de densité de peuplement qui nous est familière a été complétée par celle d'《 indice de concurrence ». En fait, ce sont deux aspects du même sujet qui diffèrent par le niveau de l'étude. C'est-à-dire, selon que l'on considère le peuplement dans son ensemble, ou bien chaque arbre forestier au milieu du peuplement.

Le présent article traite de ces travaux qui ont eu des applications diverses avec intention d'en faire ressortir l'unité pour parvenir plus facilement à des conclusions et mieux saisir la direction des perspectives. Sauf précision ils portent généralement sur des peuplements équiennes purs ayant dépassé la phase juvénile des premières années après la plantation. II est bon de rappeler que Bartet et Pleines, avaient déjà attiré l'attention sur certains d'entre eux en 1972, en pressentant leur intérêt.

\section{2. - Densité des peuplements}

\section{1. - Les problèmes posés par la définition de la densité d'un peuplement}

Curtis a analysé le terme "densité » à travers son usage courant : il en ressort que ce mot est étroitement lié aux notions de "concurrence, occupation de surface, et fermeture de couvert », qui sont les « concepts fondamentaux de la sylviculture » (Curtis, 1970).

Cette remarque cependant ne suffit pas. Lorsque l'on utilise des mesures dans l'intention de préciser des lois de croissance, il devient nécessaire de s'appuyer sur une définition qui doit permettre d'évaluer du point de vue des notions citées les conditions moyennes du développement et de la croissance des arbres d'un peuplement.

Un élément important devrait guider la recherche de cette définition : il s'agit de la possibilité de pouvoir comparer les densités de peuplements d'âges et de productivités différents, impliquant que les valeurs calculées de la densité soient indépendantes de ces caractères $\left({ }^{1}\right)$.

Ce n'est, par exemple, pas le cas de la surface ou l'espacement moyen par arbre, que l'on estime à partir du nombre de tiges à l'unité de surface, et qui ont été identifiées très naturellement à la densité. Car ces quantités n'ont de rapport avec la densité d'un peuplement, que si l'on tient compte de son âge et de la station où il se trouve $\left({ }^{2}\right)$. Cela a donc pour inconvénient de supprimer toute possibilité de distinguer entre les effets de l'âge, de la station, et de la densité sur la croissance.

C'est bien pour s'affranchir de cette dépendance que dans le facteur de Hart ou d'autres semblables, l'espace moyen est rapporté à la hauteur du peuplement. Cette façon de normaliser le nombre de tiges à l'unité de surface part du principe que tout espace moyen n'a de sens, comme espace vital, que relativement à la dimen-

(1) C'est-à-dire, telles que l'âge et la station d'un peuplement ne puissent être une indication sur des valeurs plus probables de sa densité.

$\left({ }^{2}\right)$ On pourrait ajouter aussi : de son histoire. 
sion moyenne des arbres. En gardant cette idée, on peut chercher à introduire ce caractère de relativité d'une façon plus élaborée, en faisant directement intervenir les besoins d'extension horizontale de la cime des arbres.

La mesure de densité connue Outre-Atlantique sous le nom de «Crown Competition Factor » est l'exemple d'une telle tentative.

\section{2. - Le facteur de concurrence des cimes, une mesure de densité}

L'article de Krajicek, Brinkman, Gingrich, publié en 1961 sous le titre : « La concurrence des cimes - Une mesure de densité » n'a pas cessé d'inspirer d'autres travaux ; il est à cause de cela d'un intérêt particulier. Le « facteur de concurrence des cimes » ou «CCF » (pour Crown Competition Factor) est la mesure de densité proposée par ces auteurs.

Elle est fondée sur l'observation qu'il existe, pour les arbres forestiers de chaque espèce s'étant développés sans concurrence $\left({ }^{1}\right)$, une relation entre leur diamètre à $1,30 \mathrm{~m}$ et le diamètre de leur couronne $\left({ }^{2}\right)$ (fig. 1.1$)$. Cette relation est indépendante

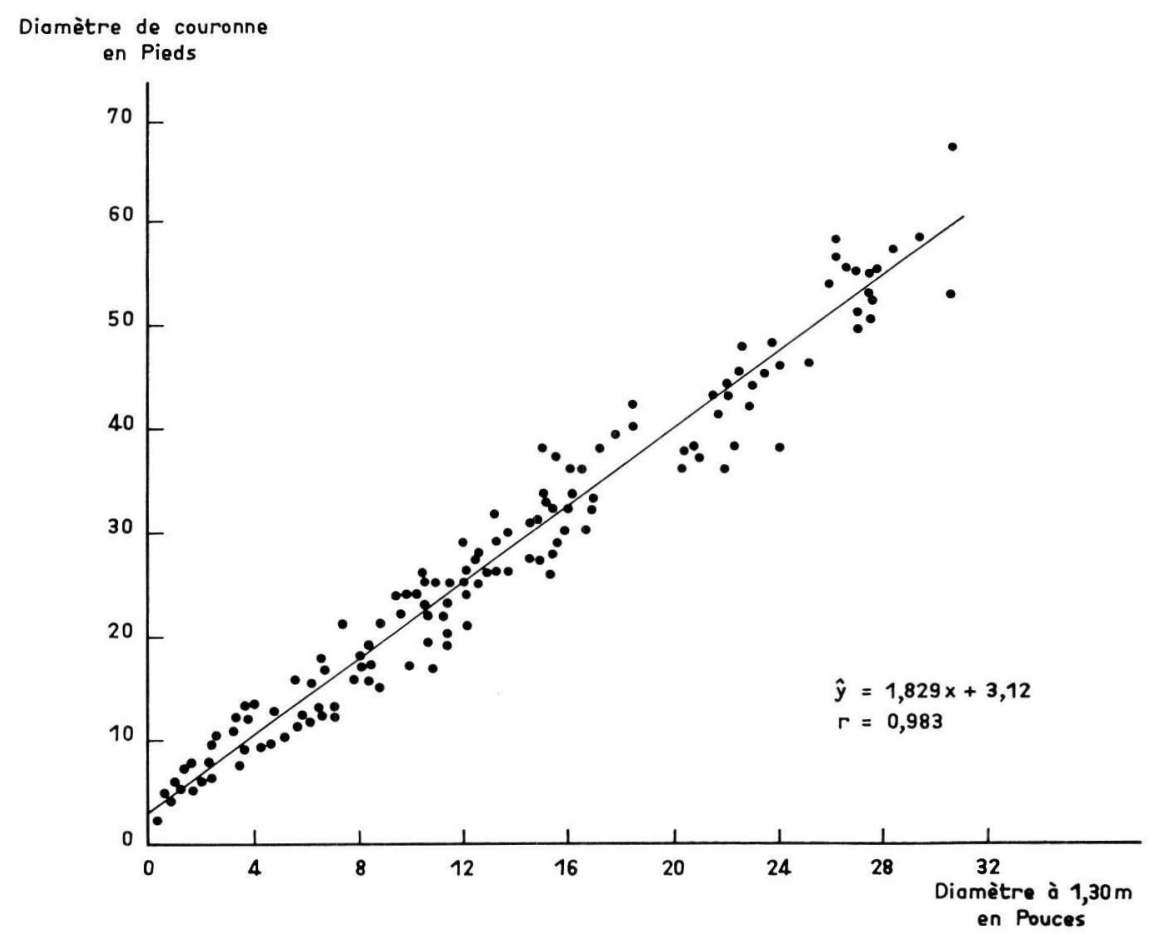

FIG. 1.1. - Relation fondamentale du CCF pour l'hickory et deux variétés de chênes (Quercus alba, Quercus velutina). Les arbres de plein champ de ces espèces, de tous âges et sur diverses stations, n'ayant pas présenté de différences, une seule régression a été calculée sur l'ensemble des données (d'après Krajicek et al., 1961).

Open-grown crown width-d.b.h. relation for combined hickory, white oak, and black oaks.

(1) Dans tout ce qui suit, ces arbres seront parfois nommés « arbres de plein champ selon une expression consacrée, et pour des raisons pratiques. Pourtant, il vaut mieux supposer que les arbres dont il est question ici se sont développés dans une certaine ambiance forestière, qui a pu les protéger de conditions climatiques extrêmes.

$\left({ }^{2}\right)$ On entend précisément par là le diamètre de la projection de cime. 
de l'âge des arbres et de la station où ils se trouvent, et du point de vue statistique, présente une faible dispersion. Elle n'est plus vérifiée pour des arbres en peuplement ; les écarts sont attribués aux effets de la concurrence, et servent à mesurer son intensité.

Pour chaque espèce on peut calculer avec la méthode des moindres carrés, deux coefficients $a$ et $b$ tels que le diamètre moyen $C W$ de la couronne des arbres de plein champ, de diamètre $D$ à $1,30 \mathrm{~m}$ soit donné par la relation :

$$
C W: a+b D \text {. }
$$

Pour tout arbre de diamètre $D($ à $1,30 \mathrm{~m}$ ), la surface moyenne maximum de couronne ou «MCA » (pour Maximum Crown Area ») est alors définie par :

$$
M C A=\frac{\pi(a+b D)^{2}}{4} .
$$

Le CCF d'un peuplement de $\mathrm{N}$ tiges supposé fermé et occupant la surface $\mathrm{A}$, est par définition, le rapport exprimé en pourcentage, de la somme des MCA des arbres du peuplement à la surface totale des projections de cime de ses arbres (assimilée à la surface du peuplement) :

$$
C C F=\frac{1}{A} \sum_{i=1}^{N}(M C A)_{i} 100=\frac{25 \pi}{A}\left(N a^{2}+2 a b \sum_{i=1}^{N} D_{i}+b^{2} \sum_{i=1}^{N} D_{i}^{2}\right) .
$$

On peut considérer que les arbres des peuplements dont le CCF est approximativement égal à 100 se sont développés sans se gêner ; au delà de cette valeur, la croissance individuelle devrait être ralentie par la concurrence et d'autant plus que le CCF est plus fort (fig. 1.2).

PLACETTES

A

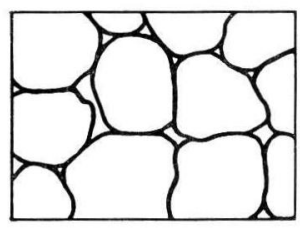

$C C F=100$

Coefficient de fermeture du couvert $=1,0$
B

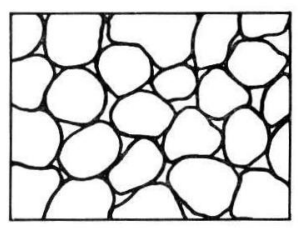

$C C F=200$

C.f.c. $=1,0$ c

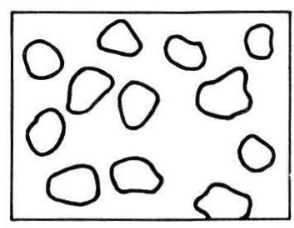

$C C F=100$

C.f.c. $=0,50$

FIG. 1.2. - Comparaison de trois placettes hypothétiques de même âge, sur la même station, mais variant par le CCF et la fermeture du couvert.

- Placette A : Les arbres se sont développés sans concurrence dans le passé, mais ont maintenant atteint le point de complète fermeture du couvert : CCF $=100$;

- Placette B : Le développement des cimes des arbres est limité par la concurrence : CCF $=200$;

- Placette C : Histoire identique à celle de la placette $B$, mais récemment éclaircie. (D'après Curtis, 1970.) 
L'étude originale de Krajicek et al. a été réalisée avec plusieurs variétés de chênes, des hickories, et des épicéas communs : au total 340 arbres ; les coefficients de corrélation dans tous les cas étaient supérieurs à 0,9. Depuis, de nombreuses relations permettant de calculer le CCF pour des peuplements d'autres essences ont été établies dans des conditions comparables $\left({ }^{1}\right)$.

Curtis (1970) a proposé d'étendre la définition du CCF à des peuplements non fermés en l'associant à une mesure de fermeture du couvert (fig. 1.2).

Ce paragraphe sera complété par des exemples d'application du CCF où apparaîtront ses propriétés.

\section{3. - Le quotient d'espace vital de Chisman et Schumacher}

Les principes du CCF présentent des similitudes avec ceux du « quotient d'espace vital » ou «TAR » (pour «Tree Area Ratio ») de Chisman et Schumacher (1940).

La définition du TAR d'un peuplement quelconque est basée sur une relation qui existe entre le diamètre à $1,30 \mathrm{~m}$ et la surface de projection de cime des arbres des peuplements naturels à peu près équiennes de la même essence, où la concurrence est extrêmement grande ; peuplements dits « normaux » aux U. S. A. et au Canada $\left({ }^{2}\right)$.

Dans ce cas, les mesures individuelles de cime ne sont pas nécessaires pour établir la relation fondamentale. Soient, en effet, a, b, c, les constantes de cette relation pour une espèce donnée. Pour chaque arbre i de surface de projection de cime $(T A)_{i}$ et de diamètre $D_{i}$ à 1,30 m dans un peuplement normal, elle s'écrit :

$$
(T A)_{i} \simeq a+b D_{i}+c D_{i}^{2} .
$$

Si l'on mesure les diamètres des $N$ arbres d'un tel peuplement sur une surface égale à l'unité de surface, on a :

$$
1 \simeq \sum_{i=1}^{N}(T A)_{i} \simeq a N+b \sum_{i=1}^{N} D_{i}+c \sum_{i=1}^{N} D_{i}^{2} .
$$

Etant donné une série de peuplements «normaux » de tous âges provenant de stations de diverses productivités, la relation (1) s'applique à chacun d'eux, et permet d'estimer les coefficients $a$, b et $c$, par la méthode des moindres carrés.

Le TAR d'un peuplement quelconque sur une surface unité est la valeur obtenue en appliquant la formule de calcul (1) à l'ensemble des diamètres à 1,30 m des arbres de cette surface ; il donne «... une estimation de la proportion d'unité de surface qui serait occupée par les arbres de la placette, si chacun d'eux était affecté de la surface moyenne occupée par un arbre du même diamètre, dans une placette normale » (Curtis, 1970a). Il est parfois exprimé en pourcentage qui s'obtient en multipliant sa valeur par 100.

\section{4. - Les diverses mesures de densité d'un peuplement}

Curtis $(1970,1971)$ a longuement discuté les mesures relatives de densité de peuplement. II s'est efforcé de rechercher parmi elles « des analogies et des équivalences »,

(1) Voir Vezina $(1962,1963)$. Par ailleurs, Van Slyke a obłenu ces relations pour seize espèces différentes, avec des coefficients de corrélation allant de 0,73 à 0,99 , avec une moyenne de 0,93 .

$\left({ }^{2}\right)$ On nomme ainsi dans ces pays des peuplements qui se sontétablis de façon naturelle, n'ont pas subi d'intervention sylvicole, et sont de densité maximum pour l'espèce considérée.

Certains peuplements témoins des expériences européennes en sont un bon exemple. 
en s'attachant à démontrer que : «... la plupart de ces mesures peuvent être considérées comme le quotient d'une surface moyenne disponible par arbre, à celle occupée par des arbres comparables en diamètre, hauteur, ou autres attributs, et dont la croissance s'effectue dans des conditions de densité («normales » ou « de plein champ ») servant de référence $)$.

D'abord guidé par des considérations théoriques (1970), Curtis a pu confirmer ses vues dans une seconde étude (1971) où diverses mesures de densité ont été calculées et comparées dans plusieurs analyses pour différents peuplements, à partir de données chiffrées provenant d'expériences d'espacement et d'éclaircies de Douglas (Pseudotsuga menziesii).

Un certain nombre de relations fondamentales dans les peuplements « normaux» de Douglas ayant été préalablement établies par des analyses de régression, les mesures suivantes furent comparées:

- Le Tree Area Ratio de Chisman et Schumacher TAR.

- La surface terrière relative $G_{0} /\left(G_{e} \mid \bar{D}\right)$ quotient de la surface terrière $G_{0}$ de la placette observée à la surface terrière $G_{e}$ d'une placette normale de même diamètre moyen $\bar{D}$ (et de même surface).

- Le nombre de tiges relatif $N_{0} /\left(N_{e} \mid H G\right), N_{0}$ désigne le nombre de tiges de la placette observée, $N_{e}$ celui d'une placette normale de même hauteur moyenne $H G$.

- La surface terrière relative $G_{0} /\left(G_{e} \mid A, S\right)$ où la référence est la surface terrière d'une placette normale de même âge $A$ et de même productivité $S$.

- La surface terrière relative $G_{0} /\left(G_{e} \mid \bar{H}_{D C D}, A\right) \bar{H}_{D C D}$ désignant la hauteur moyenne des arbres dominants et codominants.

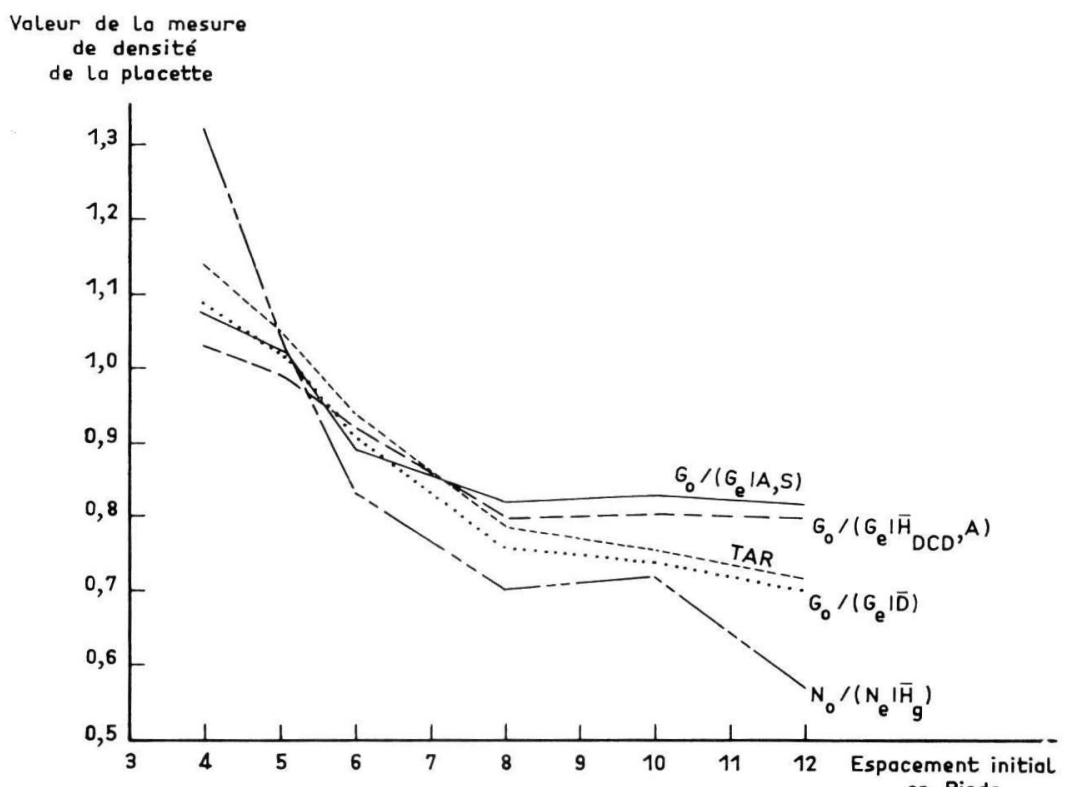

FIG. 1.3. - Comparaison de mesures de densité de peuplement sur les données de placettes d'une expérience d'espacement à l'âge de 42 ans (d'après Curtis, 1971).

Comparison of stand density measures for data of a plantation spacing test. 
En particulier, toutes ces mesures appliquées à des données provenant d'une expérience d'espacements à la plantation ont présenté pour chaque placette (à l'âge de 42 ans) des valeurs voisines, et pour l'ensemble de ces placettes à peu près même allure de variation (fig. 1.3) suivant l'espacement initial. Mais cette étude comporte de nombreux autres points d'intérêt : par exemple il y est montré que la corrélation entre les mesures en question et l'âge ou la productivité est très faible.

Enfin, dans un article publié en 1967, Gingrich a fait état d'une observation (sur deux essences feuillues) selon laquelle les relations entre le diamètre à 1,30 m et le diamètre de la couronne des arbres de plein champ, et de peuplements « normaux, sont des droites proportionnelles (fig. 1.4). II n'y a aucune raison de penser que ce fait soit particulier aux essences étudiées dans cet article, et il complète la démonstration de Curtis car le CCF et le TAR ne devraient différer que par un coefficient de proportionnalité.

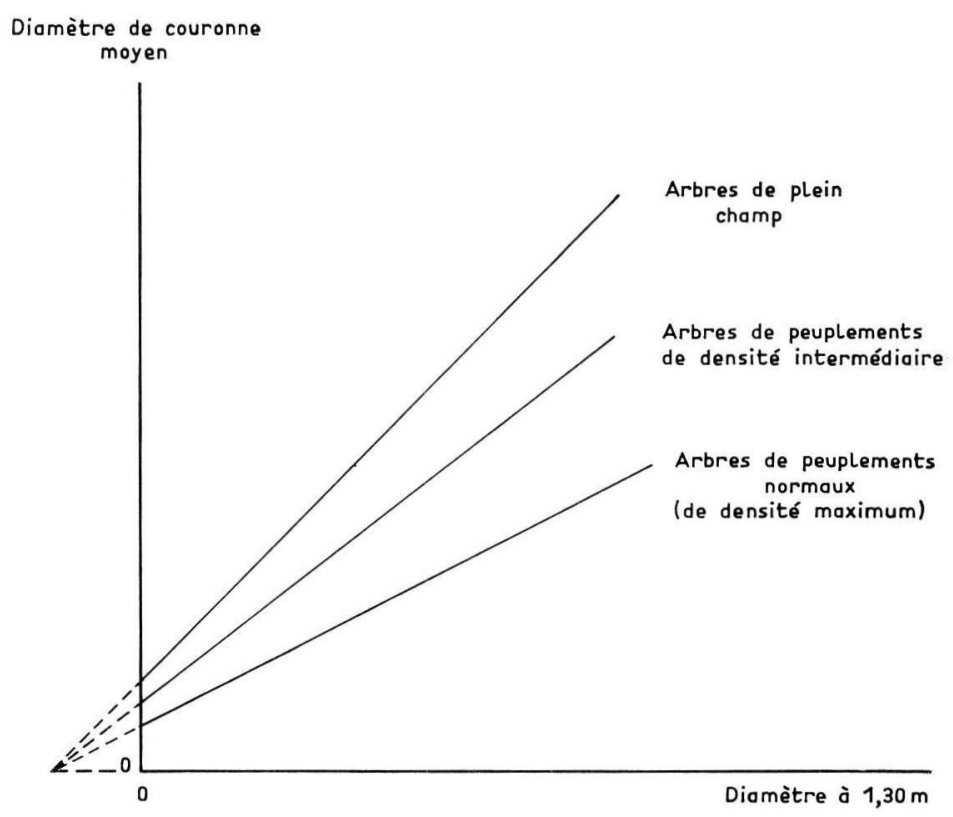

FIG. 1.4. - Relations entre le diamètre de la couronne et le diamètre à 1,30 m d'arbres provenant de peuplements de diverses densités (d'après Curtis, 1970).

Average crown width-diameter reference curves.

II est important de rappeler que toutes ces mesures ne peuvent s'appliquer qu'à des peuplements homogènes et fermés, car il est nécessaire que tout l'espace disponible soit occupé par les arbres pour que la notion de surface moyenne par arbre soit bien liée aux conditions de croissance.

\section{5. - Exemples d'application du CCF à l'étude des peuplements forestiers}

Depuis la parution de l'article de Krajicek et al., le CCF a été étudié et utilisé dans des travaux divers, aux U. S. A. et au Canada ; beaucoup plus souvent qu'une autre mesure de densité. On peut citer notamment les arłicles suivants: 
- Dahms (1966) : pour un certain nombre de placettes de Lodgepole pine (Pinus contorta Dougl.), la surface terrière et le CCF ont été successivement utilisés dans des équations de prédiction de l'accroissement en volume, où ils figurent avec l'âge et l'indice de productivité. Le CCF ne contribue pas à réduire la variance résiduelle d'autant que la surface terrière ${ }^{1}$ ). Mais il peut cependant présenter un avantage sur celle-ci en tant que mesure de densité. D'une part à cause de sa faible dépendance vis-à-vis de l'âge et de la productivité ; mais aussi parce qu'il est une mesure plus fidèle des densités élevées $\left({ }^{2}\right)$.

- Strub, Vasey, Burkhart (1975) : une étude réalisée sur le loblolly pine (Pinus Taeda L.) a fait apparaître que les courbes du diamètre moyen en fonction de l'âge, d'un échantillon de placettes, accusent une discontinuité du taux de croissance, un an après que leur CCF ait atteint la valeur 100.

- Schenk, Moore, Adams, Mahoney (1977) : un indice établi pour des placettes de sapin (Abies grandis) en peuplement mélangé, a été observé conjointement au taux de mortalité des arbres dû à un scolyte (Scolytus ventralis). L'indice est une fonction du CCF de chaque placette et de la « diversité » des espèces d'arbres qu'elle contient. II a pu alors apparaître une relation de type exponentiel entre le nombre d'arbres tués par le ravageur pendant une période de 3 ans, et cet indice $\left(r^{2}=0,82\right)$.

Parmi les applications pratiques du CCF, il a paru intéressant de détailler les deux suivantes:

a) Les normes de Gingrich $(1964,1967)$.

Les peuplements feuillus des plateaux des états du Centre aux U. S. A. sont principalement composés de l'association chêne-hickory. Le problème se posait de classer ces peuplements, de densités variées, en vue de leur aménagement.

Il fut d'abord entrepris de définir un intervalle de densités :

Pour la limite supérieure (A), des placettes de 25 à 75 ans furent installées dans les peuplements non aménagés les plus denses (peuplements «normaux ») de diverses productivité, afin d'estimer les constantes de l'équation du TAR. Leur densité moyenne fut conventionnellement fixée à 100 .

Chêne et hickory de plein champ conduisent à la même équation de base pour le calcul du CCF (voir fig. 1.1). Cette équation devait permettre de déterminer les peuplements de trop faible densité, et servir à définir la limite inférieure $B$ de ces densités.

Or, chaque valeur du CCF se trouvant à peu près égale à 60 p. 100 de la valeur correspondante du TAR, il revenait au même d'utiliser l'une ou l'autre équation comme mesure de densité. En utilisant le TAR, une abaque fut construite (fig. 1.5), permettant d'évaluer la densité d'un peuplement donné exprimée en pourcentage de la densité maximum.

Il suffit pour cela d'en connaître la surface terrière et le nombre de tiges. Cette simplification fut admise après un examen ayant montré que le TAR n'était pas très sensible à la variation de structure des peuplements (distribution des diamètres)

( $\left.{ }^{1}\right)$ L'âge, l'indice de productivité et la surface terrière « expliquent» 82,9 p. 100 de la variation ; lorsque le CCF remplace la surface terrière, ce pourcentage tombe à 74,1 p. 100.

$\left({ }^{2}\right)$ Dahms (1966) a noté cette propriété en interprétant la courbe de régression de la surface terrière en fonction du CCF : dans un intervalle des valeurs les plus élevées du CCF (250 à 370$)$, cette courbe ne varie plus de façon sensible. 
Sur l'abaque (fig. 1.5) sont représentées, dans le système de coordonnées : nombre d'arbres par acre - surface terrière par acre, les courbes sur lesquelles la densité est constante et égale à une valeur donnée entre les seuils $A$ et $B$. La courbe $C$ (établie à partir de placettes permanentes), donne la densité des peuplements pouvant atteindre le seuil $B$ en 10 ans sur les stations de productivité moyenne.

Ces normes furent bien accueillies par les forestiers travaillant sur le terrain ${ }^{(1)}$.

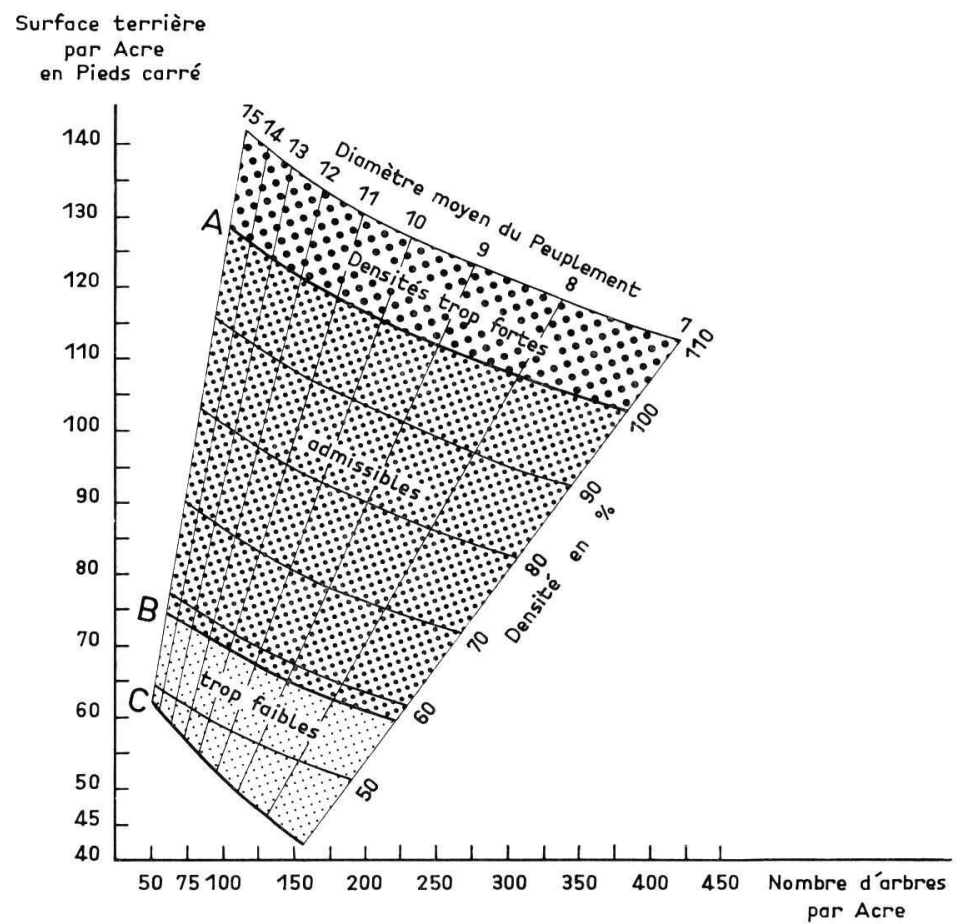

FIG. 1.5. - Relation entre la surface terrière, le nombre de tiges, le diamètre moyen, et la densité exprimée par le TAR en pourcentage de la densité maximum tolérée par les essences feuillues en mélange (principalement diverses variétés de chênes et hickories (Carya Mutt. spp.), dans les plateaux des états du Centre des U.S.A.).

La courbe $B$ représente 60 p. 100 de la densité maximum prise comme référence : c'est la densité des peuplements fermés où il n'y a pas d'effet marqué de la concurrence sur les arbres.

La courbe $C$ représente la densité des peuplements qui peuvent atteindre le niveau $B$ en 10 ans, sur des stations de productivité moyenne (d'après Gingrich, 1967).

Relation of basal area, number of trees, and average tree diameter to stocking percent for upland hardwood forests in the central states of USA.

b) Productivité, hauteur dominante ef densité, par Alexander, Tackle, ef Dahms (1967).

Le lodgepole pine aux U. S. A. est connu pour être particulièrement sensible à la densité de peuplement qui influence considérablement sa croissance en hauteur. Pour

( $\left.{ }^{1}\right)$ « Les forestiers ont pu établir les besoins sylvicoles, bien plus précisément qu'il n'était possible auparavant. Ils ont apprécié la liberté d'exercer leur art sans la restriction d'un indice rigide de densité de peuplement » (Gingrich. 1964). 


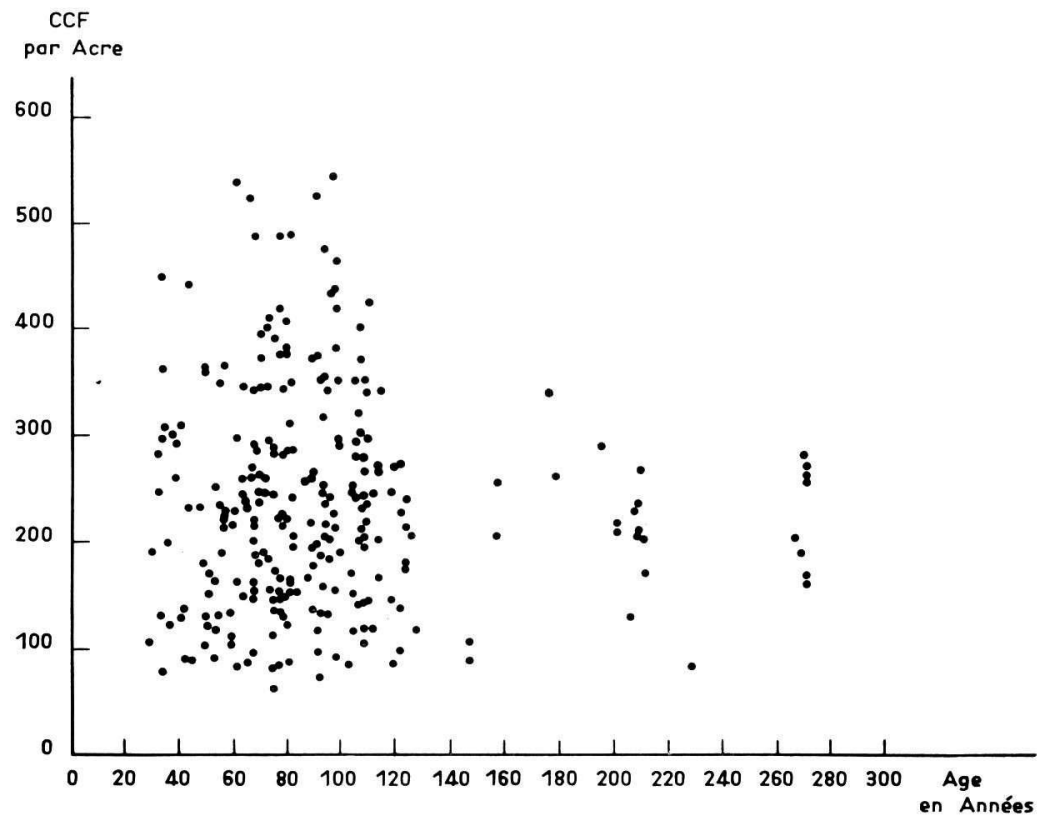

FIG. 1.6. - Relation entre le CCF ef l'âge (d'après Alexander et al., 1967). Relationship of CCF to total age.

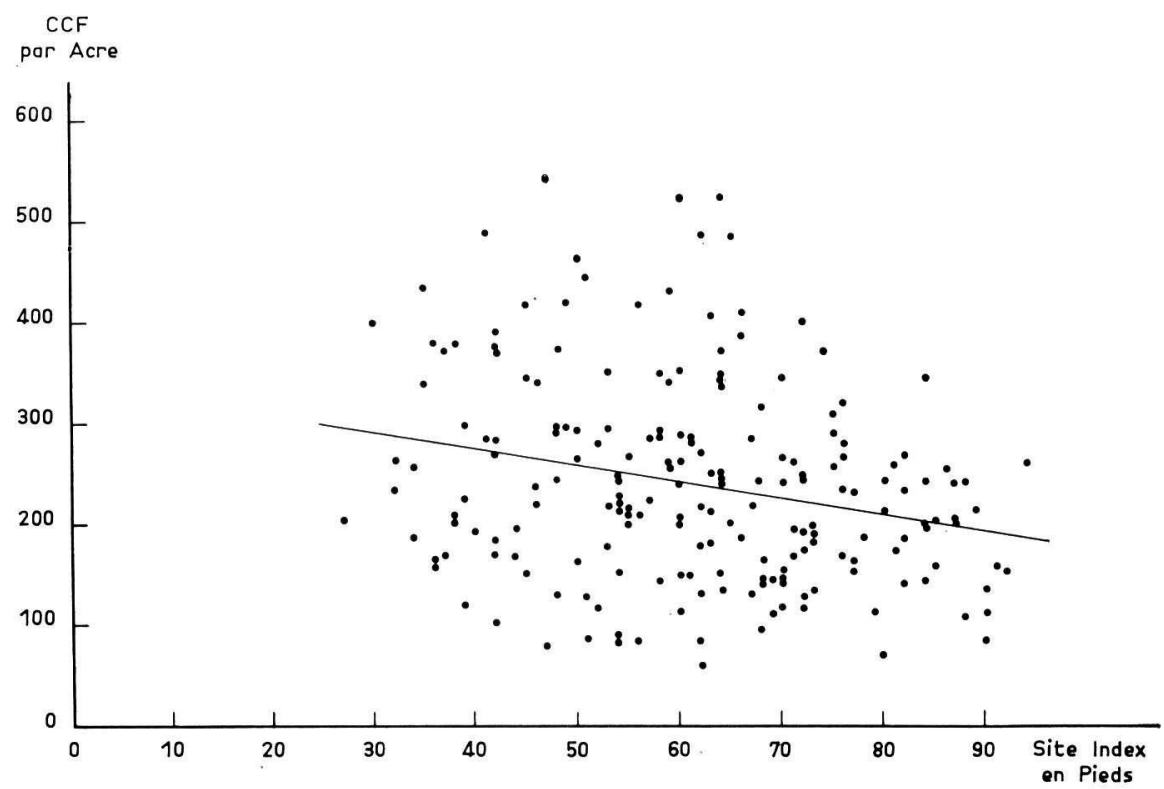

FIG. 1.7. - Relation entre le CCF et l'indice de productivité (Site Index) (d'après Alexander et al., 1967).

Relationship of CCF to site index. 
avoir le moyen de déterminer la productivité des stations de cette essence, les auteurs ont cherché à préciser comment la croissance en hauteur des arbres dépendait de la densité de peuplement.

Des systèmes de plusieurs placettes de diverses densités, installées sur chaque station, devaient permettre de réaliser cette étude. Dans chacun de ces groupes, une placette de très faible densité donnait l'indice de productivité (vrai) de la station; les autres placettes, un indice apparent suivant leur densité.

L'examen de diagrammes de dispersion (fig. 1.6 et 1.7) ayant permis d'apprécier une faible dépendance du CCF vis-à-vis de l'âge et de l'indice de productivité, cette mesure fut retenue parmi d'autres $\left(^{1}\right)$.

Ce plan d'expérience a permis d'établir principalement les résultats suivants:

1) les hauteurs dominantes étaient réduites sur toutes les stations dès que le CCF des placettes dépassait $125\left({ }^{2}\right)$,

2) la réduction de la hauteur dominante était d'autant pus importante que la productivité de la station était plus grande.

Ces résultats sont traduits par une équation de régression qui a pu être ajustée avec une bonne précision (fig. 1.8).

$$
\begin{aligned}
\mathrm{H}_{0} & =6,247+0,144(\mathrm{CCF})+1,2268(\mathrm{Si})-0,0017(\mathrm{CCF})(\mathrm{SI}) \\
\mathrm{r} & =0,99
\end{aligned}
$$

sur une base de 176 placettes

$\mathrm{H}_{\mathbf{0}}=$ hauteur dominante à l'âge de 100 ans (= site index apparent)

$\mathrm{SI}=$ indice de productivité vrai

$\mathrm{CCF}=$ crown competition factor $>1,25$.

Une relation, enfin, a pu être établie, permettant de calculer le CCF d'une placette, à partir des caractéristiques plus courantes que sont la surface terrière et le diamètre moyen (fig. 1.9).

\section{3. - Indices de concurrence}

\section{1. - Une notion de densité locale de peuplement}

Lorsque l'on veut particulariser les conditions dans lesquelles se trouve un arbre donné d'un peuplement, du point de vue de la proximité et de l'importance des autres arbres susceptibles de le gêner dans sa croissance, on introduit une notion de densité locale, qui a été formalisée par des « indices de concurrence ».

Le problème qui se pose lors de la conception d'un indice de concurrence, est celui de pouvoir caractériser les conditions locales de croissance en peuplement de chaque arbre de façon synthétique, par une valeur numérique.

A l'origine, ces recherches devaient permettre de rationaliser par la mesure certaines des appréciations fréquemment imposées au sylviculteur par son activité ;

( $\left.{ }^{1}\right)$ L'indice de productivité est ici la hauteur dominante en pieds à l'âge de 100 ans ; pour cette raison, on utilisera aussi le terme anglais «site index». Dans les diagrammes en question, le site index est donné par les placettes de faible densité servant de référence sur la station.

$\left({ }^{2}\right)$ Les auteurs signalent qu'ils sont avertis du fait que les densités de peuplement trop faibles ont aussi pour effet de diminuer la hauteur, mais cet effet n'a pas été noté dans les observations qu'ils ont effectuées. 


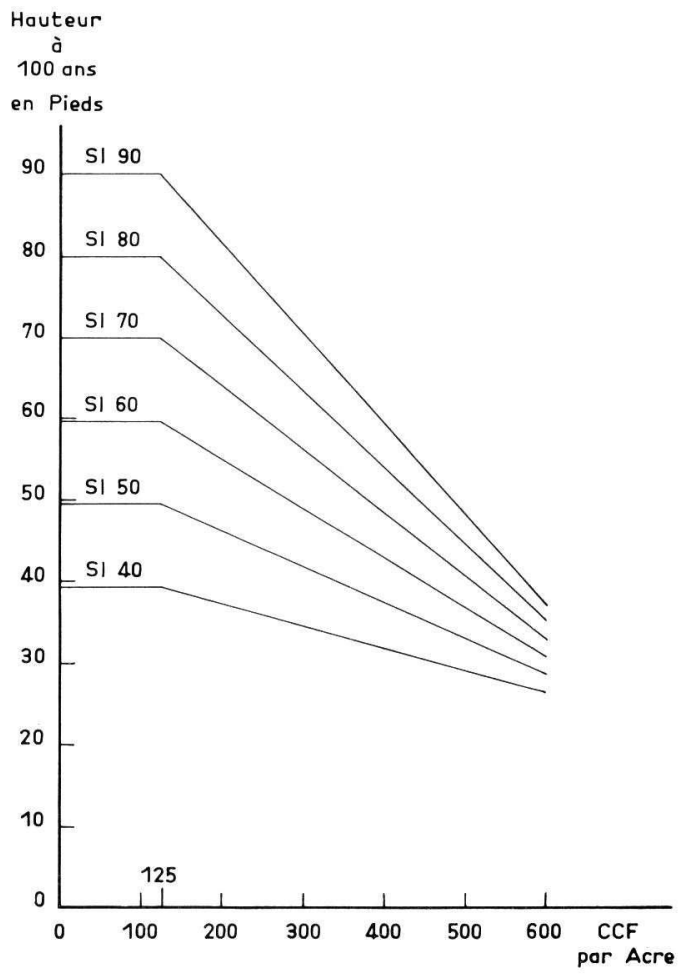

FIG. 1.8. - Relation entre la hauteur dominante à l'âge de 100 ans et le CCF pour les valeurs de l'indice de productivité comprises entre 40 et 90 , d'après l'équation (2). La croissance en hauteur n'est pas affectée par la densité au-dessous de la valeur 125 du CCF (d'après Alexander et al., 1967).

Relationship of dominant heigth at age 100 years to CCF for SI classes 40 to 90 .

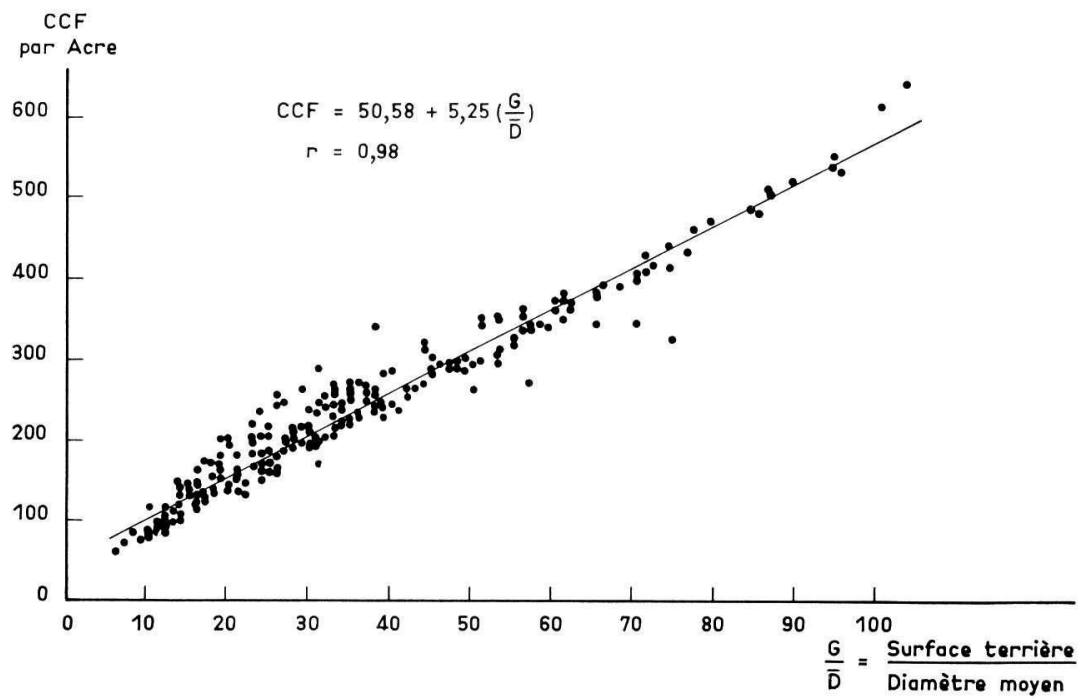

FIG. 1.9. - Relation entre le CCF et le quotient de la surface terrière par le diamètre moyen (moyenne des diamètres de la placette) (d'après Alexander et al., 1967).

Relationship of CCF to basal area/average diameter. 
elles ont eu de plus en plus pour objet de modéliser la croissance (individuelle) des arbres d'une placette forestière pour simuler son évolution sous des traitements hypothétiques. Le but n'a pas toujours été atteint, mais le bilan des recherches dans ce domaine est certainement positif.

\section{2. - Aperçu des principes classiquement développés dans l'établissement d'un indice de concurrence}

Les indices détaillés ici sont présentés à titre d'exemple. Ils ne constituent pas l'ensemble des indices qui ont pu être utilisés, mais ils donnent une bonne idée des traits principaux que l'on retrouve parmi eux.

C'est très probablement avec Staebler en 1951 qu'est apparu pour la première fois un indice de concurrence (fig. 2.1, A). Pour définir l'indice de concurrence d'un arbre en peuplement, Staebler s'appuyait sur la notion de "cercle de concurrence » reprise plus tard sous le nom de «zone d'influence ». Chaque arbre est affecté d'une zone d'influence circulaire, qui est une abstraction sensée représenter la surface maximum d'étendue horizontale qu'il pourrait utiliser (dans l'état actuel) pour sa croissance.

Les voisins d'un arbre donné sont ceux dont la zone d'influence coupe la sienne. A chacune de ces intersections est attribuée une valeur, et l'indice de l'arbre sujet à l'étude est égal à la somme de ces contributions élémentaires. La figure 2.1 expose différentes façons de mesurer les contributions élémentaires, et les indices correspondants ; la pondération par les rayons des zones d'influence qui apparaît pour la première fois chez Newnham (1964), se retrouve avec un exposant (EX.) chez Bella (1971).

Dans les indices de ce type, l'estimation du rayon de la zone d'influence d'un arbre pose un problème. La méthode qui a été généralement adoptée, fut de supposer que ce rayon était une fonction du diamètre de l'arbre, puis de prendre pour valeurs des paramètres de cette fonction, celles qui donnaient les meilleurs résultats dans une application déterminée de l'indice $\left({ }^{1}\right)$.

Suivant une voie différente et d'après une idée due à Brown (1965), Moore, Budelsky, Schlesinger (1972) ont proposé un indice dont la valeur pour un arbre donné est égale à la surface d'un polygone à l'intérieur duquel l'arbre se trouve. Chaque côté de ce polygone est perpendiculaire au segment délimité par l'arbre sujet à l'étude et un arbre adjacent, au point qui partage ce segment dans un rapport défini par les diamètres des deux arbres (fig. 2.2) (chez Brown ce rapport était fixé à 0,5).

\section{3. - Indices de concurrence et croissance individuelle des arbres en peuplement}

II y a une importante part d'arbitraire dans la définition de tels indices, mais le jugement que l'on peut porter sur ceux-ci doit être réservé jusqu'au moment où ils sont confrontés avec l'utilisation à laquelle ils sont destinés.

(1) La méthode d'Opie (1968) est particulière. Il observait, dans des placettes d'une espèce de lumière (Eucalyptus camaldulensis), des arbres plus âgés dominant une plage continue de régénération. Une dépression notée dans la hauteur moyenne de la régénération, lui permettait d'apprécier une zone d'influence circulaire autour de chaque arbre, de rayon proportionnel à son diamètre à $1,30 \mathrm{~m}$. La constante de proportionalité allait de 14 sur les meilleures stations à 17 sur les plus mauvaises. 
A

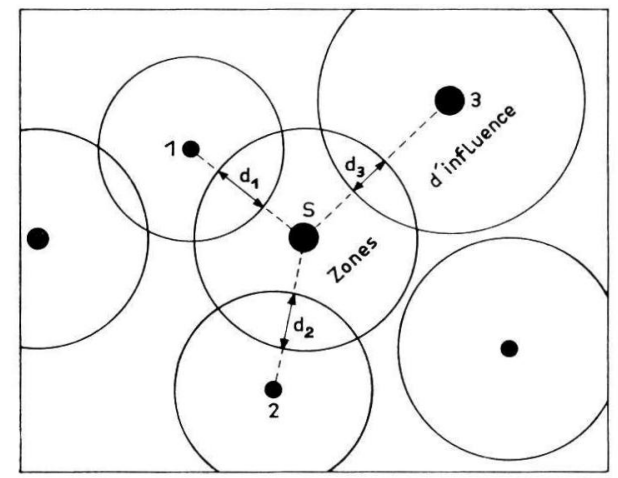

$S: C I=\sum_{i=1}^{3} d i$

(STAEBLER 1951)

B

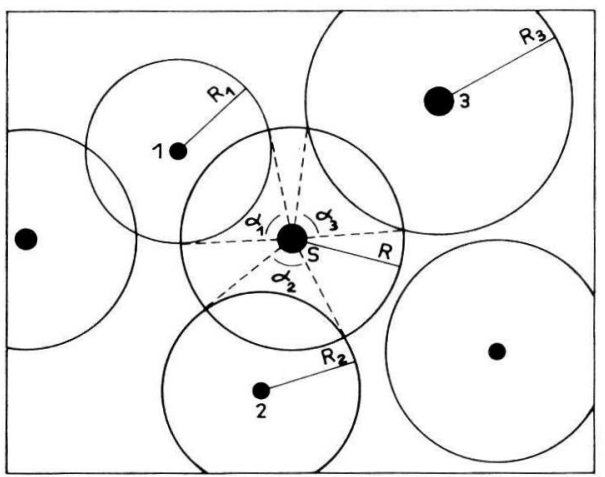

$S: C I=\frac{1}{2 \pi} \sum_{i=1}^{3} \alpha_{i} \frac{R_{i}}{R}$

(NEWNHAM

1964)

C

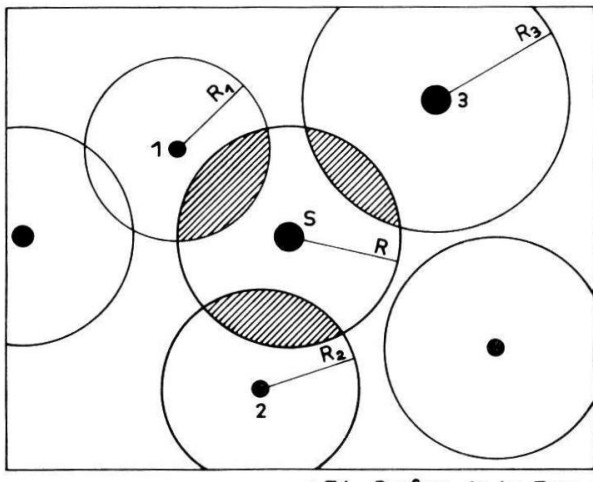

S: $C I=\frac{1}{Z A^{*}} \sum_{i=1}^{3} 20_{i}^{*}$

$\left(\begin{array}{cc}\text { (GERRARD } & 1967) \\ \text { OPIE } & 1968)\end{array}\right.$

S: CI $=\frac{1}{Z A^{*}} \sum_{i=1}^{3} Z 0_{i}^{*}\left(\frac{R_{i}}{R}\right)^{E X}$

(BELLA 1971)

- ZA Surfoce de lo Zone d'influence de $S$.

- $\mathrm{ZO}_{\mathrm{i}}$ Surfoce de L'intersection des Zones d'influance.

FIG. 2.1. - Formes diverses d'indices de concurrence basés sur la notion de zone d'influence.

L'importance de la partie de la zone d'influence de l'arbre sujet à l'étude (S), qui est recouverte par la zone d'influence d'un voisin, est évaluée de façons différentes: mesures linéaires pour Staebler, angulaires pour Newnham, de surfaces pour Gerrard, Opie, Bella.

Les pondérations des contributions élémentaires représentent l'importance relative de la partie de la zone d'influence de $S$ occupée, ou l'importance relative des deux arbres concurrents. Chez Bella. il y a un double principe de relativité.

Various types of competition indexes based on competition circle. 
De longue date, l'indice de concurrence devait fournir une information s'ajoutant à celle constituée par les dimensions de chaque arbre d'un peuplement, pour rendre compte de son accroissement observé (généralement sur le diamètre à hauteur de poitrine) pendant une courte période (de 3 à 5 ans), au cours de laquelle l'indice est supposé constant.

Les tentatives de Staebler (1951) pour relier son indice à la croissance des arbres en peuplement (Douglas) furent infructueuses, mais plusieurs auteurs s'accordent pour dire que le manque de moyens de calcul y fut pou $r$ beaucoup.

Gerrard (1967) en supposant que le rayon de la zone d'influence de chaque arbre était proportionnel à la circonférence de l'arbre, choisissait la constante qui minimisait l'erreur résiduelle dans des équations de régression « expliquant 》 l'accroissement en surface terrière par l'indice de concurrence et le diamètre des arbres. Les résultats obtenus en travaillant avec de vieux peuplements de chênes, hickories et érables en mélange, ne semblent pas avoir été probants.

Bella (1971) est le premier à avoir publié des résultats jugés satisfaisants. La méthode utilisée était similaire à celle de Gerrard, mais en plus d'une constante de proportionnalité, s'appliquant cette fois au rayon de la surface de cime maximum de l'arbre (MCA), il devait estimer un exposant (voir fig. 2.1, C). Son étude a été conduite sur des données provenant de placettes permanentes équiennes, de tremble, d'un eucalyptus, de deux pins (Pinus banksiana, Pinus resinosa) et de Douglas. Les accroissements individuels, mesurés sur des périodes allant de 4 à 5 ans dans des peuplements d'une trentaine d'années, étaient étudiés en relation avec la circonférence des arbres et leur indice de concurrence. Des effets faibles, mais significatifs de cet indice

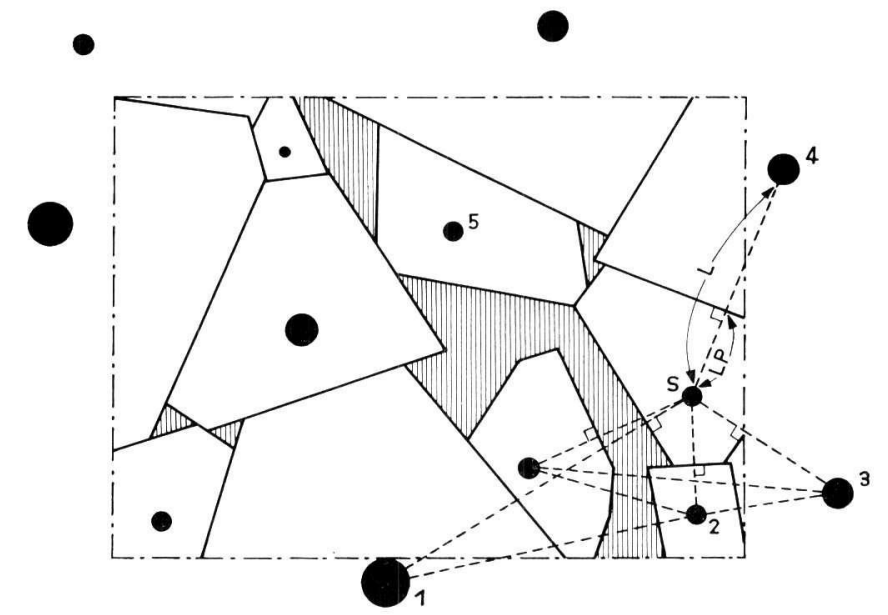

FIG. 2. 2. - L'indice de Moore et al. Chaque arbre est à l'intérieur du polygone délimité par les perpendiculaires aux segments qu'il définit avec les arbres voisins, aux points qui partagent ces segments dans les rapports $\left(D^{2} /\left(D^{2}+D_{i}^{2}\right)\right)$ où $D$ est le diamètre à hauteur de poitrine de l'arbre sujet, $D_{i}$ les diamètres des arbres voisins (d'après Moore ef al.) :

$$
\frac{\mathrm{LP}}{\mathrm{L}}=\frac{\mathrm{D}^{2}}{\mathrm{D}^{2}+\mathrm{D}_{\mathrm{i}}^{2}} \text {. }
$$

Competition index of Moore et al. 
ont pu être mis en évidence : celui-ci contribuait à réduire l'erreur résiduelle dans les équations de régression $\left(^{1}\right)$.

En reprenant la méthode de Bella, Moore et al. (1972) ont eux obtenu de meilleurs résultats avec cet indice, et des coefficients de corrélation allant de 0,7 à 0,8 . Leur indice (fig. 2.2) était simultanément étudié avec des résultats comparables. Cette fois apparaissait la hauteur dans les équations étudiées.

Enfin Lin (1974) fait état de travaux où un indice était à la base d'un modèle de croissance. Pour cet indice, des mesures angulaires étaient utilisées, un peu comme chez Newnham. Les équations utilisées font apparaître avec l'indice, ses variations causées par des éclaircies. II n'y a pas dans cet article de statistiques publiées, mais les résultats des simulations de plusieurs années de croissance de divers peuplements sont convainquants. Un examen du travail original (Lin, 1969) s'imposerait pour confirmer ce jugement.

\section{4. - Formes nouvelles d'indices de concurrence}

Récemment, un intérêt accru s'est porté sur le développement de la cime des arbres, dont l'étude apparaît de plus en plus être un facteur de progrès déterminant dans les recherches sur la croissance et la production des peuplements. Ce sujet se place parfaitement dans le cadre de ce chapitre.

Arney (1972) a utilisé un indice de concurrence défini à la hauteur de chaque verticile sur les arbres de l'espèce Douglas. Son indice (nommé CCQ) prend par défi-

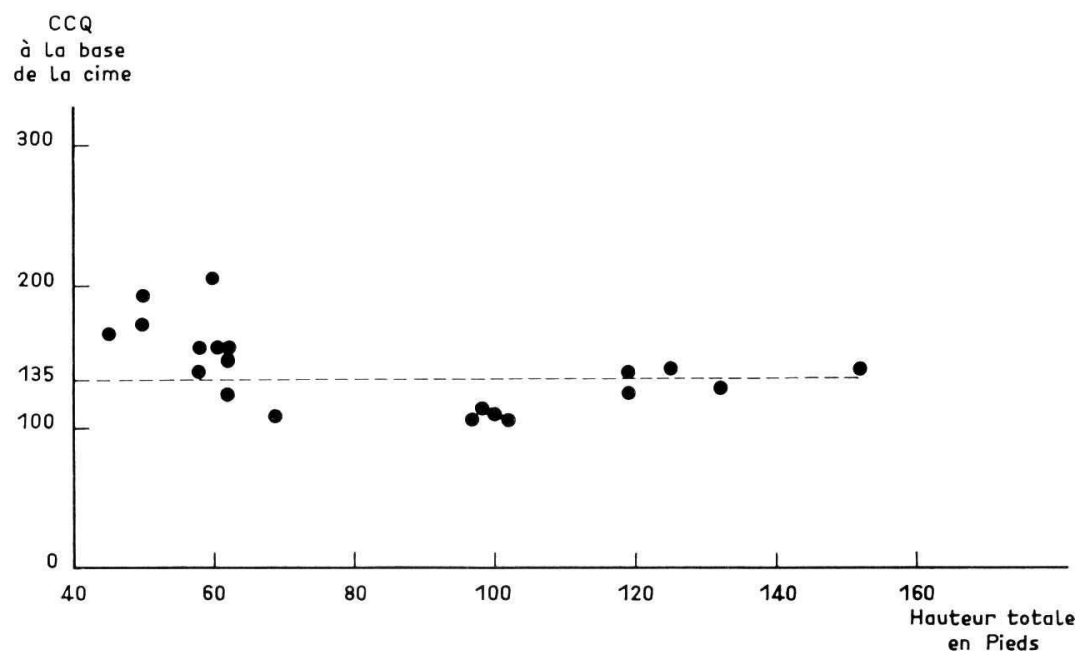

FIG. 2.3. - Arney a nommé «tolérance » (de l'espèce), la valeur de son indice de concurrence au-dessus de laquelle les cimes se nécrosent. Cette figure tend à prouver que cette valeur est indépendante de la hauteur totale des arbres, et caractéristiques de l'espèce (d'après Arney, 1972).

Tolerance as affected by total height.

(1) Il est intéressant de noter, qu'à l'issue de cette étude, ił a pu sembler à l'auteur que se confirmaient les observations d'Opie sur la variation du rayon de la zone d'influence suivant la station. D'autre part, on peut penser qu'une erreur de procédure dans l'estimation de ce rayon, a empêché l'obtention de résultats meilleurs. En effet, celui-ci était estimé en relation avec les accroissements des arbres, mais sans tenir compte de leur diamètre, contredisant ainsi un principe de relativité sur lequel l'indice est fondé (voir fig. 2.1, C). Un effeł plus marqué de l'indice aurait peut-être pu mieux se manifester avec une méthode différente. 
nition pour chaque arbre donné, la valeur 100 au-dessus du niveau où la cime entre en contact avec les cimes voisines, puis augmente régulièrement au-dessous de ce niveau ( ${ }^{1}$ ). Dans certaines conditions, Arney a pu observer une relation entre une valeur de cet indice et la hauteur de la cime vivante de l'arbre (fig. 2.3) : dans des peuplements homogènes, pour les arbres ayant atteint une certaine taille, la cime apparaît nécrosée aux niveaux où le CCQ est supérieur à 135, indépendamment de la hauteur de l'arbre.

Mais surtout, une tendance s'est affirmée, avec de bons résultats. Car depuis le CCF, et à la même époque la proposition de Czarnoswky (1961) d'évaluer la densité d'un peuplement par la moyenne des hauteurs relatives des cimes; et suivant le point de vue de Smith $\left({ }^{2}\right)$, l'idée s'est précisée de mesurer l'intensité de la concurrence par ses effets sur les cimes des arbres, avec pour corollaire le besoin de mieux connaître les lois de son développement.

Cette conception a été plus récemment illustrée par Honer (1971, 1972). Honer a d'abord constaté pour une espèce de sapin (Abies balsamea) et d'épicéa (Picea mariana) que le rayon de la cime des arbres à une distance donnée du bourgeon terminal, et pour des arbres de même hauteur, ne change pas lorsque l'on passe d'arbres croissant en plein découvert à des arbres de peuplement, si cette distance n'excède pas la hauteur de la partie supérieure de la cime libre de concurrence. Par la suite, une mesure ponctuelle de densité a été proposée, définie pour chaque individu, comme le rapport de la surface maximum de couronne permise par la hauteur de l'arbre, à la surface effective de cette couronne. C'est un indice de concurrence qui est l'équivalent, comme mesure locale de densité, de ce que représente le CCF pour les peuplements.

Dans cet ordre d'idées enfin il faut signaler les travaux de Mitchell (1975), dont l'importance est fondamentale. Mitchell a pu donner la forme la plus concrète d'un indice de concurrence avec le Douglas. Cet indice est exprimé par le rapport FV/FV $\max$ du volume réel de feuillage de chaque arbre, au volume maximum (théorique) de feuillage déterminé par la ha'steur totale de l'arbre. Pour les arbres ayant constamment bénéficié d'une croissaı ce libre, le volume $\mathrm{FV}_{\max }$ de feuillage (fixé à peu de choses près par la hauteur de l'arbre) détermine l'accroissement annuel total en volume (BI) du fût (voir fig. 2.4). Lorsqu'un peuplement se ferme, cette relation disparaît : à un même volume foliaire FV correspondent des accroissements annuels $\mathrm{BI}$ différents (fig. 2.4). Mitchell a alors montré qu'en introduisant le rapport FV/FV $\max$ qui précise le statut concurrentiel de chaque arbre, on peut à nouveau déterminer l'accroissement $\left({ }^{3}\right) \mathrm{BI}$. II faut noter, que dans cette étude, il ne s'agit plus d'une équation

${ }^{1}$ ) Cet indice de concurrence est, à peu de choses près celui de Gerrard ou Opie ; le diamètre à 1,30 m est remplacé pour chaque arbre par le diamètre à la hauteur considérée. Une relation entre la distance au bourgeon terminal et le diamètre à cette hauteur d'une part, le diamètre de la cime d'autre part, établie pour les arbres hors peuplement, permet le calcul de l'indice.

$\left({ }^{2}\right)$ Smith (1962) : «L'analyse du développement des cimes peut établir les limites économiques et biologiques à la croissance des arbres et des peuplements. »)

$\left({ }^{3}\right)$ Le volume foliaire FV est à peu de chose près le volume occupé par la frondaison.

Les volumes $\mathrm{FV}_{i}$ correspondant à chacune des 5 années successives d'aiguilles présentes sur l'arbre sont pondérés par des coefficients représentant leur efficience photosynthétique relative : $F V=\Sigma \alpha_{i} F V_{i}$.

La relation entre l'accroissement annuel et le volume foliaire s'écrit:

$$
\mathrm{BI}=0,0001012 \mathrm{FV}^{1,29}\left(1-\log \left(\mathrm{FV} / \mathrm{FV}_{\max }\right)\right)^{1,287} \text { en } \mathrm{m}^{3}
$$

(équation ajustée à partir de données provenant de 43 arbres, sur les logarithmes des deux membres : $r=0,94$ ).

Pour pouvoir intégrer ces relations à un modèle de croissance de peuplement, les lois de croissance et de développement de la cime des arbres ont du être déterminées. A cette occasion, les observations déjà citées de Honer (1971) ont été indépendamment confirmées. 
représentant quelques années isolées de croissance, mais que celle-ci s'applique à des arbres de tous âges, toutes tailles, et toutes conditions de peuplement sur une station de productivité donnée.

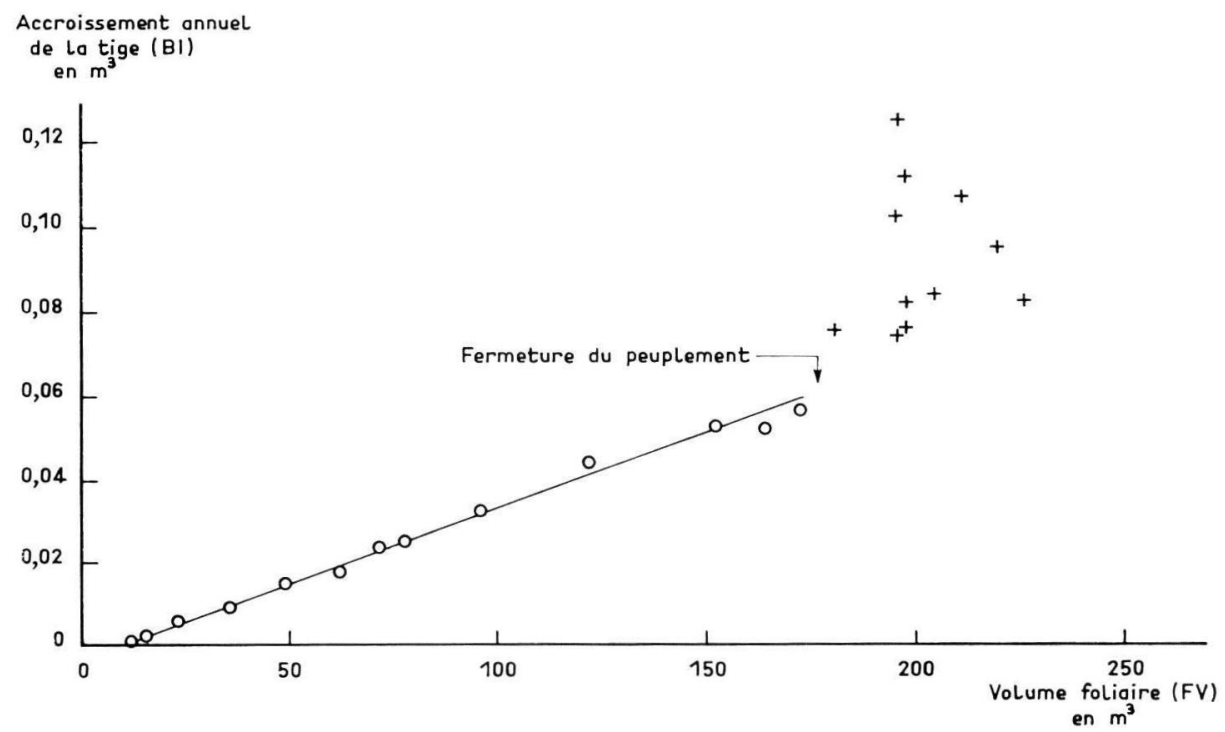

FIG. 2.4. - Relation entre l'accroissement (annuel) en volume, et le volume foliaire d'un échantillon d'arbres d'âges indéterminés dans différentes conditions de peuplement. Les valeurs sont présentées comme si un même peuplement avait été échantillonné au cours du temps (d'après Mitchell, 1975).

Relationship between Foliar volume and bole increment of a sample of trees.

\section{4. - Remarques générales et conclusion}

\section{1. - Remarques}

En ce qui concerne les mesures relatives de densité de peuplement on peut pour simplifier retenir la conclusion de Curtis (1970a) qui voyait dans le choix de l'une ou de l'autre : «... une question d'information disponible, de facilité de calcul... plutôt que des différences fondamentales dans le sens ou la précision ».

Pourtant, d'après l'exemple de circonstances où il a pu être utilisé avec succès dans des applications variées, lorsqu'une certaine finesse d'étude expérimentale est requise, le CCF pourrait parfois donner de bien meilleurs résultats que tout autre mesure de densité, grâce à sa conception qui permet de tenir compte à la fois de la composition des peuplements, de caractères importants de l'espèce considérée, et des effets cumulés de la concurrence sur les arbres des peuplements $\left(^{1}\right)$.

En ce qui concerne les indices de concurrence, une différence importante entre eux n'a pas encore été signalée. Car certains s'expriment de façon absolue (ceux de Staebler et de Moore et al.), les autres soutiennent un principe de relativité (l'indice de

(1) Il en serait probablement de même pour toute autre mesure de densité similaire basée sur l'évaluation d'un effet de la concurrence sur les cimes comme par exemple la mesure proposée par Kzarnowsky (1961) qui a été brièvement évoquée dans la section 3.4. Un avantage du CCF est, entre autres, d'avoir été éprouvé. 
Gerrard et Opie par exemple). Les premiers sont donc corrélés avec la dimension des arbres, ce qui doit être tout à fait indésirable dans certaines applications.

Les applications les plus facilement remarquées de ces indices se rencontrent dans les modèles de croissance de peuplement où l'arbre sert d'unité de croissance ${ }^{1}$ ), et plus particulièrement dans la brillante monographie de Mitchell (1975). Mais ils ne doivent pas être considérés comme restreints à ce cadre, et peuvent très probablement apporter une aide précieuse à l'étude de tout dispositif de surface réduite (comme l'ont suggéré occasionnellement Le Tacon, Oswald, Tomassone, 1969) en rendant possible l'interprétation de mesures individuelles de telle façon qu'elle puisse être étendue aux peuplements.

Un effort reste à faire pour améliorer les indices de conception simple, qui pourraient rendre ce service. Le fait que l'on puisse identifier certains travers de conception ou de méthode les concernant, permet d'augurer qu'ils sont encore perfectibles.

\section{2. - Conclusion}

Les recherches visant à bien formuler la notion de « densité » pour l'appliquer à l'étude de la croissance des peuplements ont pu donner les meilleures expressions de la densité chaque fois qu'elles se sont penchées sur la croissance et le développement individuel des arbres. Ces mêmes mesures de densité ont montré leur utilité et leur efficacité dans des applications, en apportant des progrès sensibles dans l'étude de la croissance des peuplements forestiers.

Pour poursuivre cette étude il semble indispensable de pouvoir tenir compte de la façon dont les arbres occupent et utilisent l'espace. Et la question couramment posée sur la production de bois destiges, doit être étendue au développement des cimes avec lequel elle se trouve en rapport étroit, pour que l'on puisse vraiment disposer des éléments de la réponse.

La façon de procéder et le degré de finesse de cette analyse dépendent bien sûr des circonstances. Mais l'ensemble des informations que nous possédons maintenant sur la croissance et le développement des arbres forestiers, à divers niveaux d'intégration, qu'elles soient de simples relations « diamètre de couronne - diamètre à 1,30 m », ou parfois schémas plus précis de la croissance des branches (Mitchell, 1969, 1975, Honer, 1971, 1972), permettent de penser que dans chaque cas ces études peuvent dès lors être conduites dans de bonnes conditions.

La meilleure façon de résumer l'essentiel de cette conclusion et d'introduire une définition « moderne » de l'un des objets de la sylviculture revient à Curtin (1970) :

«A n'importe quel moment du développement d'un peuplement, la dimension de couronne la plus favorable à une croissance individuelle maximum, sera rarement optimale pour une croissance maximum du peuplement. Les interactions entre la taille et la forme des arbres individuels et des couronnes, et la structure ef la densité des placettes forestières, seront importants dans toute tentative de réconcilier la croissance et le développement, à la fois des arbres et des peuplements ».

Reçu pour publication en février 1978.

(1) Selon les termes de Bartet et Pleines (1972). 


\section{Appendice}

\section{I. - Glossaire}

Ces quelques brèves définitions des variables caractéristiques des peuplements forestiers, sont rappelées pour faciliter la lecture de ce texte aux personnes qui ne sont pas accoutumées aux symboles et au vocabulaire utilisé. D'autres termes et des définitions éventuellement plus précises pourront être trouvés chez Decourt, Lemoine (Ann. Sci. forest., 1969, 26 (1), 3-44).

G Surface terrière du peuplement : somme des surfaces de section à 1,30 $\mathrm{m}$ des arbres du peuplement $\left(\mathrm{m}^{2} / \mathrm{ha}\right)$

HG Hauteur moyenne du peuplement: moyenne des hauteurs des arbres du peuplement $(m)$

$\mathrm{H}_{0} \quad$ Hauteur moyenne des 100 plus gros arbres à l'hectare du peuplement. Difficile à utiliser, cette définition est souvent adaptée. La croissance en hauteur des arbres forestiers est généralement peu sensible à la densité ; cette variable est très souvent caractéristique de la productivité de la station pour l'essence considérée $(m)$

V Volume du peuplement. C'est en fait le volume des tiges du peuplement. II est généralement très bien déterminé par les variables $G$ et HG, d'où l'importance de ces deux caractères $\left(\mathrm{m}^{3}\right)$

$\mathrm{N} \quad$ Nombre de tiges du peuplement à l'hectare.

\section{II. - Conversions}

1 pied $=0,3048 \mathrm{~m}$

1 pouce $=2,54 \mathrm{~cm}$

1 acre $=0,404686$ ha.

$$
\text { III. - Statistiques }
$$

1) Un très élémentaire rappel de certaines bases sur lesquelles se fonde la décision statistique : au cours d'une expérience, une hypothèse $H_{0}$ est formulée, et elle conduit à un calcul qui permet d'évaluer quelle probabilité avaient d'être effectuées sous l'hypothèse $\mathrm{H}_{0}$, les observations résultant de l'expérience. Si cette probabilité est «faible », l'hypothèse est rejetée.

2) Coefficient de corrélation (d'une régression. Noté $R^{2}$ ou $r^{2}$ ). Il s'exprime par :

$$
r^{2}=\frac{\sum_{i=1}^{n}\left(\hat{Y}_{i}-\bar{Y}\right)^{2}}{\sum_{i=1}^{n}\left(Y_{i}-\bar{Y}\right)^{2}}
$$

où n est le nombre des observations $Y_{i}$, estimées par $\widehat{Y}_{i}$.

C'est un nombre sans dimension qui permet d'apprécier le degré de dépendance des observations $Y_{i}$ relativement aux variables « explicatives». 


\title{
Summary
}

\author{
Density measures for trees growth studies in forest stands
}

Numerous scientific works published since the early sixties by American foresters in the U.S.A. and Canada, were motivated by the thought that an adequate expression of stand density measure should considerably help growth and yield studies.

The most widely used measure, the Crow Competition Factor, has been successfully applied to various fields such as stocking standards, true site index determination, or preventing engravercaused mortality of grand fir in mixed stands.

Closely related to stand density measures, competition indexes should be regarded as point density measures; they have long been studied in the U.S.A., but have recently found their best expression and shown their efficiency in the development of single-tree distance dependent growth models of forest stands.

In each case, stand density measures or competition indexes, it appears that good knowledge of the growth and development of individual trees is a factor of progress in forest growth and yield studies.

The studies presented in this paper are not merely speculative, and are all based on experimental investigations. The methods are generally deductive rather than inductive.

\section{Références bibliographiques}

ALEXANDER R. R., TACKLE D., DAHMS W. G., 1967. Site indexes for lodgepole pine, with correction for stand density. U.S. Forest Service Research paper, RM-29, 18 p.

ARNEY J., 1972. Computer simulation of Douglas-fir tree and stand Growth. Ph. D. Thesis. Oregon State University, $79 \mathrm{p}$.

BARTET J. H., PLEINES W. E., 1972. Revue des méthodes proposées pour la prospective et la planification à long terme en matière forestière. J. For. Switz., 21 (23 bis), 609-626.

BELLA I. E., 1971. A new competition model for individual trees. Forest. Sci., 17 (3), 364-372.

BROWN G. S., 1965. Point density in stems per acre. New Zealand Forestry Research Notes, $\mathrm{n}^{\circ} 38,11 \mathrm{p}$.

CURTIN R. A., 1970. Dynamics of tree and crown structure in eucalyptus Obliqua. Forest Sci., 1970, 16 (3), 321-328.

CURTIS R. O., 1970. Stand density measures : an interpretation. Forest. Sci., 16 (4), $403 . .414$.

CURTIS R. O., 1971. A tree area power function and related stand density measures for Douglasfir. Forest. Sci., 17 (2), 146-159.

CZARNOWSKY M. S., 1961. Dynamics of even-aged Forest stands. Louisiana State University Press, 1961, 132 p. (partiellement exploité).

DAHMS W. G., 1966. Relationship for lodgepole pine volume increment to Crown Competition Factor, basal area, and site index. Forest. Sci., 12 (1), 74-82.

GERRARD D. J., 1967. Competition quotient : an index of the competitive stress affecting individual forest trees. Ph. D. Thesis Michigan State University, 65 p.

GINGRICH S. F., 1964. Criteria for measuring stocking in Forest stands. Proceedings, Society of American Foresters, Denver, Colorado, 1964, 198-201.

GINGRICH S. F., 1967. Measuring and evaluating stocking and stand density in Upland hardwood forests in the Central States. Forest. Sci., 13 (1), 38-53.

HART H. M. F., 1928. Stambal en dunning Medd. V.h. Proefstation voor net boschwesen, zl. 219 p. (non consulté, cité par Pardé, 1961).

HONER T. G., 1971. Crown shape in open and forest grown Balsamfir and black Spruce. Canad. J. Forest. Res., 1971, 1, 203-207.

HONER T. G., 1972. A height-density concept and measure. Canad. J. Forest., Res., 2, 441-447.

HUMMEL F. C., 1954. The definition of thinning treatments. Proceedings of the 2nd Congress of I.U.F.R.O., Rome, 1953, 582-588.

KRAJICEK J. E., BRINKMAN K. A., GINGRICH S. F., 1961. Crown competition, a measure of density, Forest. Sci., 7 (1), 35-42.

LE TACON F., OSWALD H., TOMASSONE R., 1969. Etude d'un dispositif " mono-arbre » sur épicéa adulte. $3^{\circ}$ Colloque International sur l'Etude de la Production Forestière. Pragues 1969, 197-213. 
LIN J. Y., 1969. Crowing space index and stand simulation of young western hemlock in Oregon. Unpublished thesis. Duke University. 182 p. (non consulté).

LIN J. Y., 1974. Stand growth simulation models for Douglas-Fir and western hemlock in the Northwestern United States, in : J. Fries (Ed.). Growth models for tree and stand simulation. Dep. Forest Yield Res., Royal Coll. For, Stockholm, Res. Notes 30, 102-118.

MITCHELL K. J., 1969. Simulation of the growth of even-aged stands of white spruce. Yale University : School of Forestry, Bull. no 75, 48 p.

MITCHELL K. J., 1975. Dynamics and simulated yield of Douglas-fir. Forest. Sci. Mogr., 17, 39 p.

MOORE J. A., BUDELSKY C. A., SCHLESINGER R. C., 1973. A new index representing individual tree competitive status. Canad. J. Forest. Res., 3, 495-500.

NEWNHAM R., 1964. The development of a Stand model for Douglas-fir. Ph. D. Thesis. University of British Columbia, 201 p.

OPIE J. E., 1968. Predictability of individual tree growth using various definitions of competing basal area. Forest. Sci., 14 (3), 314-323.

PARDÉ J., 1961. Comment préciser l'intensité d'une éclaircie. Revue Forestière Française, aoûtseptembre 1961, pp. 551-557.

SCHENK J. A., MOORE J. A., ADAMS D. L., RONALD L. N., 1977. A preliminary hazard rating of gran fir stands for mortality by the fir engraver. Forest. Sci., 23 (1), 103-110.

SMITH G. H. G., 1963. Analysis of crown development can establish biological and economic limits to growth of tress and stands. Commonwealth Forestry Rev., 42 (1), 27-33.

STAEBLER G. R., 1951. Growth and spacing in an evenaged stand of Douglas-fir. Unpublished thesis. University of Michigan. 46 p. (non consulté, cité par Gerrard, 1967).

STRUB M. R., VASEY R. B., BURKHART H. E., 1975. Comparison of diameter growth and Crown Competition Factor in loblolly pine plantations. Forest. Sci., 21 (2), 427-431.

VAN SLYKE A. L., 1964. An evaluation of crown measures for coniferous trees and stands. Univ. of Brit. Columbia, Faculty of Forestry, 1964, 50 p. (non consulté, cité par Gingrich, 1964).

VEZINA P. E., 1962. Crown width-d.b.h. relationship for open-grown balsam fir and white spruce in Quebec. Forestry Chron., 38, 463-473.

VEZINA P. E., 1963. More about the "crown competition factor ». Forestry Chron., 39, 313-317.

VEZINA P. E., 1963. Objective measures of thinning grades and methods. Forest. Chron., 39 (3), 290-299. 